\title{
ORIGINAL
}

\section{RELACIÓN DEL NIVEL DE ACTIVIDAD FÍSICA, PRESIÓN ARTERIAL Y ADIPOSIDAD CORPORAL EN ADOLESCENTES MADRILEÑOS}

\author{
Carlos Alberto Cordente Martínez (1), Pilar García Soidán (2), Manuel Sillero Quintana (1) y \\ Jesús Domínguez Romero (1) \\ (1) Facultad de Ciencias de la Actividad Física y el Deporte. Universidad Politécnica de Madrid. \\ (2) Facultad de Ciencias Sociales y de la Comunicación. Universidad de Vigo.
}

\section{RESUMEN}

Fundamento: La creciente preocupación por la salud ha suscitado un enorme interés en el estudio de los factores que inciden sobre ella. El objetivo de este trabajo es analizar la influencia del nivel de actividad física (NAF) en la salud de los adolescentes y determinar su relación con la presión arterial (PA) y la composición corporal.

Métodos: La investigación se realizó entre noviembre de 2002 y febrero de 2003 utilizando el Cuestionario Modificable de Actividad Física para Adolescentes que mide el nivel de actividad física. También se midieron la composición corporal y la presión arterial, en una muestra compuesta por 554 adolescentes de 35 centros educativos de Madrid, con edades comprendidas entre 12 y 18 años.

Resultados: Resultaron inactivos el 13,2\% de los varones y el 36,4\% de las mujeres. Los promedios para las PA sistólica/diastólica fueron de $125,6 / 71,3 \mathrm{mmHg}$ en los varones y 118/69,4 $\mathrm{mmHg}$ en las mujeres, pero sólo se detectó influencia del nivel de actividad física en la PA diastólica para los varones. Presentaron tendencia al sobrepeso el 48,27\% de las mujeres frente al 13,53\% de los hombres. En el caso de las mujeres se encontró relación entre adiposidad corporal y nivel de actividad física.

Conclusiones: El nivel de actividad física se presenta como un factor asociado a la salud de los adolescentes, si bien se manifiesta de forma distinta en cada sexo. En los hombres afecta significativamente a la PA diastólica, mientras que en las mujeres su influencia se detecta en el grado de adiposidad corporal.

Palabras clave: Adolescentes. Conducta del adolescente. Presión arterial. Composición corporal.

\section{Correspondencia:}

Carlos Alberto Cordente Martínez

Facultad de Ciencias de la Actividad Física y del Deporte. C/ Martín Fierro s/n.

28008 Madrid

Correo electrónico: carlos.cordente@upm.es

\section{ABSTRACT}

\section{Relationship of the Degree of Physical Activity, Blood Pressure and Body Fat among Teenagers in Madrid}

Background: It is throughout childhood and adolescence that many behavior patterns which are going to have a powerful influence on health during adult life are established. This study is aimed at analysing the influence physical activity has on the health of adolescents and at determining the relationship thereof with blood pressure (BP) and body composition.

Methods: The research was conducted from November 2002 to February 2003, using the Modifiable Physical Activity Questionnaire for Adolescents, which measures the degree of physical activity. The anthropometric variables and blood pressure were also measured for the sample comprised of 554 adolescents within the $12-18$ age range from 35 schools in Madrid.

Results: A total of $13.2 \%$ of the males and $36.4 \%$ of the females were found to be inactive. The averages for the systolic/diastolic BP were $125.6 / 71.3 \mathrm{mmHg}$ in the males and $118 / 69.4 \mathrm{mmHg}$ in the females, but physical activity was found to have an influence solely on the diastolic BP for the males. A total of $48.27 \%$ of the females showed a tendency toward being overweight, as compared to $13.53 \%$ of the males. In the case of the females, a relationship was found to exist between body fat and degree of physical activity.

Conclusions: The degree of physical activity shows itself to be a factor related to the health of adolescents, although taking on a different focus in each gender. Among the males, it has a significant bearing on the diastolic BP, whilst among the females, its influence is seen in the degree of body fat.

Key words: Adolescents. Adolescent Behavior. Body composition. Blood pressure. Exercise. 


\section{INTRODUCCIÓN}

En las últimas décadas diversos estudios científicos han demostrado que la actividad física regular proporciona importantes beneficios en la salud ${ }^{1-5}$. En España estamos asistiendo durante los últimos años a un creciente interés en el análisis de las relaciones entre la actividad física y la salud. En ello ha jugado un papel fundamental el incremento de las enfermedades cardiovasculares, el apoyo que ha recibido la medicina preventiva para reducir los costes de la medicina curativa y la extensión de un concepto más abierto y dinámico de la salud, que se ha orientado a la promoción de ambientes y estilos de vida saludables ${ }^{3}$.

Es a lo largo de la infancia y adolescencia cuando se instauran muchas pautas de comportamiento que van a tener una influencia poderosa sobre la salud en la vida adulta ${ }^{2,6,7}$. Los estudios científicos han puesto en evidencia los riesgos que acarrea la vida sedentaria en el desarrollo de enfermedades coronarias, obesidad, hipertensión, diabetes, cáncer de colon y cáncer de mama $^{8}$. Aunque estas enfermedades se manifiesten clínicamente durante la edad adulta, su proceso puede originarse en la infancia 9 .

De los estudios revisados en relación al NAF de los adolescentes, la gran mayoría señalan valores preocupantes dada la edad de los sujetos de estudio ${ }^{5,6,10-12}$.

En cuanto a la hipertensión arterial (HTA), hoy en día representa un problema y un reto para la salud pública en nuestro país pues existe una alta prevalencia de la misma: en torno al $35 \%$ de la población adulta, el $43 \%$ entre los 35 y 65 años y el $56 \%$ en personas de 60 años o más ${ }^{13}$. Esta enfermedad tiene una serie de repercusiones sociales que afectan tanto a la esfera individual del enfermo como a la familiar y laboral. La HTA también tiene grandes repercusiones económicas en cuanto al gasto asistencial del enfermo (hospital y ambulatorio), gasto farmacéutico y gasto ocasionado tanto por muertes prematuras (el 25\% del total de muertes y el $40 \%$ de las debidas a enfermedades cardiovasculares en España) como por las diferentes discapacidades que ocasiona esta enfermedad. Entre 1995 y 2001 en España, el gasto en antihipertensivos se incremento un $61,7 \%$ suponiendo en 2001 un 14,3\% del gasto farmacéutico total con cargo al sistema nacional de salud (SNS). Por tanto este dato no incluye, el consumo intra-hospitalario, el consumo privado o a cargo de otras entidades aseguradoras, ni el consumo de medicamentos sin receta o excluidos de la financiación por el $\mathrm{SNS}^{14}$.

La terapia para hipertensos se compone a menudo de una intervención farmacológica e incluso higiénica, que incluye reducción de la toma de sal, reducción del peso corporal e incremento de la condición aeróbica.

Sin embargo, son varios los trabajos $^{10,15,16}$ que ponen de manifiesto que, entre niños y adolescentes, el NAF no influye en los valores de la PA. Este resultado contradice el de otros estudios realizados en otras franjas de edad ${ }^{3,17,18}$ pero no excluye un efecto protector de la actividad física en la PA en edades más avanzadas.

Con respecto a la composición corporal, la cantidad relativa de tejido adiposo (porcentaje de grasa) es la medida más común en adultos y niños debido a la relación bien establecida entre masa grasa, condición física, rendimiento atlético y alteraciones endocrinas y nutricionales ${ }^{19}$.

La obesidad y el sobrepeso son de gran interés para la sanidad pública, a causa de su asociación con el aumento de riesgo para la hipertensión, diabetes, enfermedades coronarias, dislipemia, cálculos biliares, algunos cánceres, etc. ${ }^{20,21}$. La preocupación de la salud pública se ha extendido a la población pediátrica, en la cual la frecuen- 
cia de la obesidad está aumentando alarmantemente en los países desarrolla$\operatorname{dos}^{7,20,22,23}$. La obesidad en los adolescentes está relacionada significativamente con la obesidad adulta y a los factores de riesgo coronario $^{24}$. Factores ambientales como la sobrealimentación o la inactividad física han sido identificados como las causas principales de la obesidad infantil ${ }^{20,25}$.

El objetivo de este trabajo es determinar el NAF de los adolescentes de la ciudad de Madrid y analizar su relación con dos parámetros de salud: la PA y la composición corporal.

\section{MATERIAL Y MÉTODOS}

Se trató de recoger datos de los distintos distritos de Madrid así como de diferentes tipos de centros educativos (públicos y privados) y, por tanto, abarcar adolescentes de diferentes niveles socio-económicos. Finalmente, se tomaron datos de 35 centros educativos elegidos al azar, 18 públicos y 17 privados, de 16 de los 21 distritos de Madrid. En cada centro se midieron sujetos de $2^{\circ}, 3^{\circ}$ y $4^{\circ}$ de ESO seleccionados aleatoriamente. Los alumnos de $1^{\circ}$ de ESO fueron eliminados tras un estudio piloto por las dificultades que mostraban para completar el trabajo en el tiempo necesario. Se entregaron 2646 cartas para los padres o tutores de los adolescentes, consiguiéndose firmas de conformidad para 1622 alumnos $(61,3 \%)$, entre los cuales se eligieron aleatoriamente a los participantes en el estudio (578) cuantificándose 554 encuestas válidas $(95,8 \%)$, correspondientes a 266 varones y 288 mujeres. Debe destacarse que, en el estudio de la PA, tan sólo cumplieron los criterios de la $\mathrm{OMS}^{26}$ de estandarización en su medición 204 varones y 270 mujeres. Por ello, teniendo en cuenta el universo al que nos referimos (44041 varones y 45258 mujeres matriculados en $2^{\circ}, 3^{\circ}$ y $4^{\circ}$ de ESO en el Municipio de Madrid durante el curso 2002-2003), para el estudio del NAF y de la composición corporal se obtuvo un error muestral de $\pm 5,6 \%$ para el conjunto del Municipio de Madrid, con la excepción del estudio de la PA donde el error muestral fue de $\pm 6,9 \%$, en todos los casos al nivel de confianza del 95,5\%. La tabla 1 muestra la distribución de los sujetos estudiados por edades y género respectivamente.

La recogida de datos se realizó entre los meses de noviembre de 2002 y febrero de 2003. A cada sujeto participante en el estudio, previa autorización de sus padres o tutores, se le encuestó y se le realizaron medidas antropométricas y de PA siguiendo un principio de garantía total del anonimato de los datos aportados. Las mediciones antropométricas y de presión arterial fueron realizadas por dos únicos sujetos, un varón y una mujer, licenciados en Ciencias de la Actividad Física y el Deporte (CCAF y D),

Tabla 1

Distribución de los sujetos de la muestra por edad (años cumplidos) y género

\begin{tabular}{|c|c|c|c|c|c|c|c|}
\hline & & \multicolumn{4}{|c|}{ Género } & \multirow{2}{*}{\multicolumn{2}{|c|}{ Total }} \\
\hline & & \multicolumn{2}{|c|}{ Varones } & \multicolumn{2}{|c|}{ Mujeres } & & \\
\hline & & $\mathbf{N}$ & $\%$ & $\mathbf{N}$ & $\%$ & $\mathbf{N}$ & $\%$ \\
\hline \multirow{7}{*}{ Edad } & 12 & 1 & $0,2 \%$ & 3 & $0,5 \%$ & 4 & $0,7 \%$ \\
\hline & 13 & 60 & $10,8 \%$ & 57 & $10,3 \%$ & 117 & $21,1 \%$ \\
\hline & 14 & 79 & $14,3 \%$ & 94 & $17,0 \%$ & 173 & $31,2 \%$ \\
\hline & 15 & 80 & $14,4 \%$ & 83 & $15,0 \%$ & 163 & $29,4 \%$ \\
\hline & 16 & 36 & $6,5 \%$ & 32 & $5,8 \%$ & 68 & $12,3 \%$ \\
\hline & 17 & 9 & $1,6 \%$ & 15 & $2,7 \%$ & 24 & $4,3 \%$ \\
\hline & 18 & 1 & $0,2 \%$ & 4 & $0,7 \%$ & 5 & $0,9 \%$ \\
\hline \multicolumn{2}{|c|}{ Total } & 266 & $48,0 \%$ & 288 & $52,0 \%$ & 554 & $100,0 \%$ \\
\hline
\end{tabular}


debidamente entrenados para esta tarea. Cada uno tomó los datos a los sujetos de su mismo género. La encuesta, auto-administrada, fue supervisada por una tercera persona, igualmente licenciada en CCAF y D y preparada para ello.

Para medir el NAF de los sujetos de la muestra se utilizó el Cuestionario Modificable de Actividad Física para Adolescentes ${ }^{27}$ que previamente fue traducido y validado para su utilización en España ${ }^{28}$. Teniendo en cuenta el Amsterdam Longitudinal Growth And Health Study [ALGHS] ${ }^{29}$ y siguiendo las recomendaciones realizadas desde la English Health Education Authority (HEA) y el American College of Sport Medicine (ACSM), se ha utilizado la siguiente clasificación del NAF :

$$
\begin{aligned}
- & =49 \mathrm{MET} / \mathrm{semana}=\text { Activo } / \text { Muy } \\
& \text { activo. } \\
- & 28-49 \mathrm{METs} / \mathrm{semana}=\text { Moderada- } \\
& \text { mente activo. } \\
- & <28 \mathrm{METs} / \mathrm{semana}=\text { Inactivo } / \text { Seden- } \\
& \text { tario. }
\end{aligned}
$$

Con respecto a la medición de la PA, con el fin de evitar los sesgos del observador, se decidió utilizar aparatos electrónicos semiautomáticos (Omron 705CP) validados ${ }^{30}$ conforme a los criterios de la American Association of Medical Instrument y de la British Hypertension Society. Como resultado definitivo se utilizó el promedio de 2 lecturas válidas de la PA. Conforme a los valores de referencia propuestos para niños y adolescentes españoles por el Grupo Cooperativo Español para el Estudio de los Factores de Riesgo Cardiovascular en la infancia y adolescencia en España ${ }^{31}$, se consideraron los valores de PA sistólica y/o diastólica para que un sujeto pueda ser considerado hipertenso, los valores que superaran el percentil 95 para su edad y género.

En cuanto a la medición del porcentaje de grasa corporal, se siguieron las recomenda- ciones del International Working Group of Kinanthropometry que son las seguidas por el Grupo Español de Cineantropometría $[\mathrm{GREC}]^{32}$. Se registraron los siguientes pliegues de grasa subcutánea: tríceps, subescapular, suprailíaco, abdominal, muslo anterior y pierna medial. Las mediciones fueron realizadas con un calibre de grasa de la compañía Holtain Ltd. (Dyfed, Reino Unido) que es el recomendado por el Internacional Biological Programme ${ }^{33}$.

Para el cálculo del porcentaje de AC utilizamos las ecuaciones de Carter $^{34,35}$.

$$
\begin{aligned}
& \text { Varones: \% } \mathrm{AC}=0,1051 * \sum 6 \text { pliegues }+2.585 \\
& \text { Mujeres: } \% \mathrm{AC}=0,1548 * \sum 6 \text { pliegues }+3.580
\end{aligned}
$$

La clasificación de los sujetos según su nivel de AC se hizo en base a los criterios del Manual de Cineantropometría de la Federación Española de Medicina Deportiva ${ }^{35}$.

En lo que se refiere al tratamiento de la información recopilada, se diseñó una aplicación en formato Microsoft Excel para la grabación de datos, que luego fueron depurados y analizados utilizando el programa estadístico SPSS 11.5, concluyéndose el estudio en Septiembre de 2005. En primer lugar se llevó a cabo un análisis univariante, con objeto de describir cada variable por separado, que fue complementado con el estudio de asociaciones entre variables. Para esto último se aplicó el test chi-cuadrado de independencia bivariante, por simplicidad, o el test ANOVA, si se verificaba el supuesto de normalidad, trabajando en cada caso al nivel de confianza del $95 \%$.

\section{RESULTADOS}

\section{Nivel de actividad física}

Considerando la totalidad de la muestra, se observa que el $45,1 \%$ de los sujetos tiene un NAF alto o muy alto mientras un $25,3 \%$ 
son inactivos o sedentarios. Además, separando por sexo y con la clasificación indicada anteriormente, se concluye que existe una diferencia significativa $\left(\mathrm{chi}^{2}=79,91\right.$; $\mathrm{p}=0,000)$ en el NAF de varones y mujeres, como se presenta en la figura 1 , obteniéndose como valores extremos que el $64,2 \%$ de los varones son activos o muy activos mientras el $36,4 \%$ de mujeres son inactivas o sedentarias.

Figura 1

Distribución de los sujetos de la muestra según nivel de actividad y género

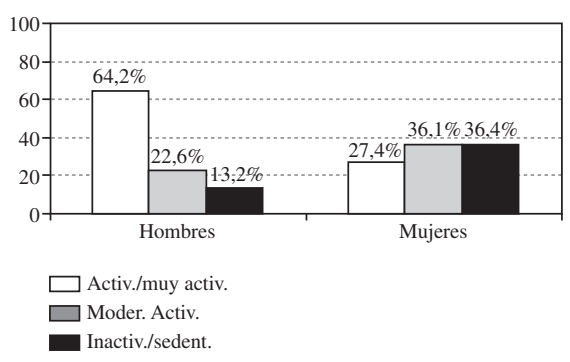

\section{Presión arterial}

Aplicando el test ANOVA se detecta la influencia del factor sexo en la PA sistólica $(\mathrm{F}=36,48 ; \mathrm{p}=0,000)$, presentando ésta niveles inferiores y menos dispersos para las mujeres, no pudiéndose extender dicha conclusión a la PA diastólica $(\mathrm{F}=3,59$; $\mathrm{p}=0,058)$. De hecho, se han obtenido los valores promedios de 125,6/71,3 $\mathrm{mmHg}$ entre los varones y $118 / 69,4 \mathrm{mmHg}$ entre las mujeres para las presiones sistólica/diastólica, respectivamente, con errores muestrales de $0,95 / 0,78 \mathrm{mmHg}$ para los varones y de 0,83/0,67 mmHg para las mujeres. Además, se observó que un $11,5 \%$ de las mujeres y un $11,8 \%$ de los varones de la muestra tienen hipertensión sistólica (HTAS) y un $4,4 \%$ de las mujeres y un $9,8 \%$ de los varones tienen hipertensión diastólica (HTAD). Los sujetos con hipertensión tanto sistólica como diastólica son el 2,2\% de las mujeres y un $1,5 \%$ de los varones.
En cuanto a la relación entre el NAF y la PA, el test ANOVA sólo permite aceptar la relación de la PA diastólica en el caso de los varones $(\mathrm{F}=5,41 ; \mathrm{p}=0,021)$, obteniéndose intervalos al 95\% de confianza del orden de 67,9-72 mmHg para la PA diastólica de los alumnos activos o muy activos, o de 71,276,9 mmHg para los demás casos.

\section{Nivel de adiposidad corporal}

Tal y como podemos observar en la figura 2, al aplicar el test ANOVA, se puede concluir la diferencia significativa existente en el nivel de adiposidad corporal de varones y mujeres ( $\mathrm{F}=704,34 ; \mathrm{p}=0,000)$, obteniéndose valores promedios respectivos de 11,15 y 20,47 en porcentaje de adiposidad corporal y errores muestrales correspondientes del orden de 0,25 y 0,24. La clasificación de los sujetos de la muestra, conforme a la propuesta realizada en el Manual de Cineatropometría $^{38}$ de la Federación Española de Medicina Deportiva, se resume en la tabla 2. Sin embargo, hemos de señalar que los resultados expuestos pueden contener un cierto sesgo debido a que no incluyen a las 10 mujeres con un sobrepeso evidente que no pudieron incluirse en el estudio por negarse a ser medidas y/o pesadas.

En base a la clasificación mencionada, se deduce también la relación significativa del

Figura 2

$$
\begin{gathered}
\text { Diagramas de caja del porcentaje de adiposidad } \\
\text { corporal en función del género }
\end{gathered}
$$

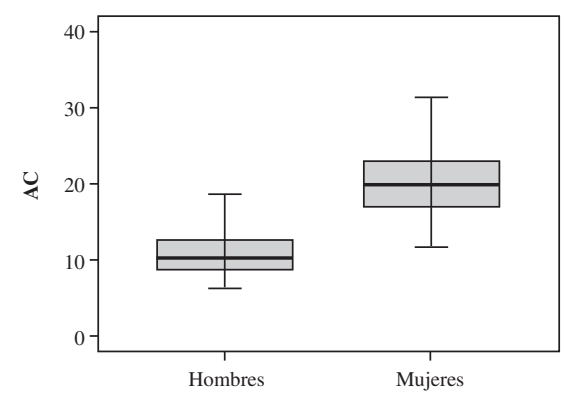


Tabla 2

Niveles relativos de peso corporal en función del nivel de adiposidad corporal en los varones y mujeres de la muestra

\begin{tabular}{|c|c|c|c|c|c|c|}
\hline \multirow{2}{*}{ AC } & \multicolumn{2}{|c|}{ Todos } & \multicolumn{2}{|c|}{ Hombres } & \multicolumn{2}{|c|}{ Mujeres } \\
\hline & $\mathrm{N}^{\circ}$ casos & $\%$ & $\mathrm{~N}^{\circ}$ casos & $\%$ & $\mathbf{N}^{\circ}$ casos & $\%$ \\
\hline Delgado/a & 34 & 6,14 & 29 & 10,90 & 5 & 1,74 \\
\hline Óptimo & 345 & 62,27 & 201 & 75,56 & 144 & 50,00 \\
\hline Lig. Sobrep. & 123 & 22,20 & 32 & 12,03 & 91 & 31,60 \\
\hline Sobrepeso & 47 & 8,48 & 4 & 1,50 & 43 & 14,93 \\
\hline Obeso/a & 5 & 0,90 & 0 & 0,00 & 5 & 1,74 \\
\hline Total & 554 & 100,00 & 266 & 100,00 & 288 & 100,00 \\
\hline
\end{tabular}

\section{Figura 3}

Distribución de las mujeres de la muestra por nivel de actividad física en función del nivel de adiposidad corporal

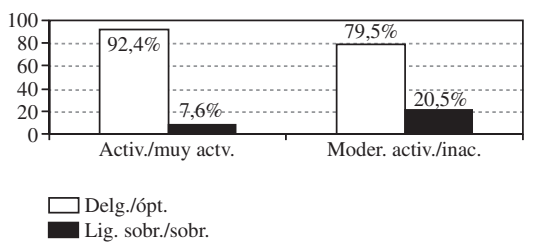

NAF con el nivel de AC en el caso de las mujeres $\left(\mathrm{chi}^{2}=21,159\right.$; $\left.\mathrm{p}=0,007\right)$, aumentando la tendencia al sobrepeso cuando la actividad física es a lo sumo moderada, como se aprecia en la figura 3.

\section{DISCUSIÓN}

Los resultados expuestos anteriormente se refieren exclusivamente a estudiantes del Municipio de Madrid. No obstante, esto no debería suponer un obstáculo a la hora de extender los mismos al conjunto de los adolescentes de ese Municipio, si se tiene en cuenta el número de sujetos participantes en el estudio y la forma en que se ha realizado el muestreo. Por otra parte, conviene señalar las limitaciones de índole metodológica a las que se exponen este tipo de estudios debido a la falta de objetividad en la medición del NAF; sin embargo, la mayoría de autores consideran aceptable su utilización en adolescentes y adultos debido a que facilitan la viabilidad de los estu$\operatorname{dios}^{2,9}$.

También cabe reseñar la escasez de estudios realizados con datos de adolescentes españoles, como la muestra que se obtiene y utiliza en esta investigación, lo cual ha motivado que en algunos casos las comparaciones se lleven a cabo recurriendo a estudios foráneos.

Podemos señalar, como hecho más destacable que, de los estudiantes de $2^{\circ}$ a $4^{\circ}$ de ESO del Municipio de Madrid de este estudio, un $25,3 \%$ tiene un NAF inferior a lo recomendable si se considera el total de la muestra. Aún así, estos resultados no son tan preocupantes como los obtenidos en

Tabla 3

Resumen de los resultados sobre el nivel de actividad física obtenidos en el trabajo de Cantera y Devís (2000) y en el presente trabajo

\begin{tabular}{|c|c|c|c|c|c|c|}
\hline \multirow{2}{*}{ Estudio } & \multicolumn{3}{|c|}{ Varones } & \multicolumn{3}{c|}{ Mujeres } \\
\cline { 2 - 7 } & $\begin{array}{c}\text { Activos y } \\
\text { muy activos }\end{array}$ & $\begin{array}{c}\text { Mod. } \\
\text { activos }\end{array}$ & $\begin{array}{c}\text { Inactivos y } \\
\text { sedentarios }\end{array}$ & $\begin{array}{c}\text { Activas y } \\
\text { muy activas }\end{array}$ & $\begin{array}{c}\text { Mod. } \\
\text { activas }\end{array}$ & $\begin{array}{c}\text { Inactivas y } \\
\text { sedentarias }\end{array}$ \\
\hline Teruel 1997 & $45,2 \%$ & $23,4 \%$ & $30,3 \%$ & $21,2 \%$ & $24 \%$ & $50,3 \%$ \\
\hline Madrid 2003 & $64,2 \%$ & $22,6 \%$ & $13,1 \%$ & $27,4 \%$ & $36,1 \%$ & $36,4 \%$ \\
\hline
\end{tabular}


una muestra de 367 adolescentes de Teruel $^{10,36}$, resumidos en la tabla 3 .

También aportan mejores resultados que los encontrados en el estudio realizado por Pate, Heath, Dowda y Trost $^{5}$ sobre una muestra representativa de adolescentes norteamericanos $(14,1 \%$ de sujetos sedentarios frente a un $22,8 \%$ muy activos), en el trabajo sobre adolescentes norteamericanos de zonas rurales de Savage y $\operatorname{Scott}^{11}$ (32\% de adolescentes activos frente al 33,2\% de inactivos o sedentarios) o en los resultados obtenidos por Van Mechelen, Twisk, Berheke Post, Snel y Kemper en el ALGHS ${ }^{29}$ (32,8\% de sujetos inactivos).

Sin embargo, las conclusiones del presente trabajo no son tan optimistas como las citadas en la revisión de Aaron y Laporte ${ }^{37}$ de la "Youth Risk Behavior Survey", la "National Children and Youth Survey" y el "Adolescent Injury Control Study", que indican que tan sólo un 10,4\% de los adolescentes son inactivos frente al $63,7 \%$ de sujetos activos.

El hecho de alcanzar el 25,3\%, de sujetos inactivos o sedentarios en la muestra observada de adolescentes madrileños, constituye un valor preocupante en estas edades por las repercusiones que pueda tener en la salud a medio y largo plazo, más si tenemos en cuenta que después de la adolescencia el NAF tiende a caer de forma importante tanto entre varones como entre mujeres $^{22}$. Otro punto a destacar es que la diversidad en la metodología empleada en
Tabla 4

Resumen de los resultados sobre el nivel de actividad física de adolescentes holandeses (1981), europeos (2001) y madrileños (2003). A los sujetos con actividad física moderada se les considera activos

\begin{tabular}{|l|c|c|c|c|}
\hline \multirow{2}{*}{ Estudio } & \multicolumn{2}{|c|}{ Varones } & \multicolumn{2}{c|}{ Mujeres } \\
\cline { 2 - 5 } & Activos & Inactivos & Activas & Inactivas \\
\hline Holanda 1981 & $69,35 \%$ & $30,65 \%$ & $64,05 \%$ & $35,95 \%$ \\
\hline Europa 2001 & $81,90 \%$ & $18,10 \%$ & $62,00 \%$ & $38,00 \%$ \\
\hline Madrid 2003 & $86,8 \%$ & $13,10 \%$ & $63,50 \%$ & $36,40 \%$ \\
\hline
\end{tabular}

la medición de la actividad física y la clasificación de los sujetos hace difícil las comparaciones con otros estudios; por ello, proponemos la unificación de criterios en este sentido entre los estudiosos de la actividad física y sus determinantes.

$\mathrm{Al}$ igual que en la gran mayoría de estudios revisados, encontramos diferencias muy claras en el NAF en ambos sexos $2,9,10,29,36,38-44$. Sin embargo, nuestros resultados entre las mujeres no son tan dramáticos como los encontrados entre las mujeres italianas de 14 a 18 años donde encontraron un $8 \%$ de mujeres activas y un $65 \%$ de inactivas o sedentarias ${ }^{12}$.

A la vista de todos estos resultados, sugerimos que la realización de campañas de promoción de la actividad física y del deporte puede ser necesaria para la población general pero, muy especialmente, en el caso de la población femenina.

Como ya comentamos anteriormente, nos parece especialmente interesante comparar nuestros resultados con el ALGHS ${ }^{29}$,

Tabla 5

Resumen de los resultados sobre la presión arterial (en $\mathrm{mmHg}$ ) obtenidos en diferentes estudios

\begin{tabular}{|l|c|c|c|c|c|}
\hline \multicolumn{1}{|c|}{ Autores } & Lugar & Año & $\begin{array}{c}\text { Edades } \\
\text { (años) }\end{array}$ & $\begin{array}{c}\text { Presión arterial } \\
\text { Varones }\end{array}$ & $\begin{array}{c}\text { Presión arterial } \\
\text { Mujeres }\end{array}$ \\
\hline Paulus et al. & Bélgica & 1999 & $12-17$ & $125 / 74$ & $122 / 74$ \\
\hline V. Mechelen et al. & Holanda & 2000 & $13-16$ & $126,6 / 76,1$ & $122,1 / 73,2$ \\
\hline Twisk et al.* & Holanda & 2002 & 32 & $135 / 86$ & $125 / 83$ \\
\hline Boreham et al. & Irlanda & 2002 & $12-15$ & $118,5 / 76$ & $107,5 / 71,5$ \\
\hline Cordente et al. & Madrid & 2003 & $13-17$ & $125,6 / 71,3$ & $118 / 69,4$ \\
\hline
\end{tabular}

(*) El estudio de Twisk et al. (2002) es la continuación longitudinal del estudio de Van Mechelen et al. (2000). 
ya que utilizaron los mismos criterios que nosotros para clasificar el NAF de los adolescentes. Exponemos en la tabla 4 los valores medios del NAF obtenidos en este estudio con sujetos de entre 13 y 16 años. Un aspecto importante a señalar es que estos datos fueron tomados en 1981. Asimismo, tal y como lo podemos observar en la tabla 4 , resulta muy interesante la comparación de nuestros datos con el "European Youth Heart Study"43, ya que sus datos fueron obtenidos mediante métodos objetivos en 2001 en sujetos de 15 años de cuatro países europeos: Dinamarca, Portugal, Estonia y Noruega.

Observamos una gran similitud en los tres trabajos en lo que respecta al NAF de las mujeres, mientras que parece que los adolescentes europeos varones actuales son claramente más activos de lo que lo eran en Holanda en 1981.

En lo que se refiere a la PA, podemos ver en la tabla 5 la comparación con nuestro estudio de los valores medios encontrados en los diferentes trabajos revisados. En relación a la tasa de hipertensión tanto sistólica como diastólica observada $(2,2 \%$ de las mujeres y $1,9 \%$ de los varones), nuestros resultados son inferiores al 11,4\% encontrado en una muestra de Bélgica de características similares a la nuestra ${ }^{45}$. Con Soler, Gil y Rey ${ }^{46}$ coincidimos en que es mayor la prevalencia de la HTAS que de HTAD (11,6\% y 6,8\% de los sujetos, respectivamente).

Podemos añadir que nuestros resultados coinciden parcialmente con aquellos estudios que no encuentran relación alguna entre el NAF y la HTA en ninguno de los dos $\operatorname{sexos}^{4,37}$. Esta ausencia de relación entre ambas variables apoyaría la idea de que los efectos de la práctica de actividad física o de su ausencia o escasez en los factores de riesgo de enfermedad cardiovascular se manifiesten habitualmente a más largo plazo. Por tanto, parece que no tiene mucho sentido sugerir nuevos estudios de la asociación entre NAF y PA si no es en sujetos de mayor edad o en el marco de un estudio longitudinal.

En lo que respecta a la diferencia significativa encontrada entre géneros en el nivel de $\mathrm{AC}$, los resultados obtenidos concuerdan en todos los estudios similares ${ }^{47}$, si bien en lo que se refiere a la relación establecida entre el NAF y la AC para las mujeres las conclusiones coinciden totalmente con algunos estudios ${ }^{3}$ y sólo parcialmente con otros que también encuentran esta asociación entre los varones ${ }^{4}$.

Aunque el sobrepeso y la obesidad pueden deberse a causas genéticas y/o metabólicas, esto es así en muy pocos $\operatorname{casos}^{47}$. El sobrepeso y obesidad suelen estar originados, principalmente, por incorrecto balance energético producido por una alta ingesta alimenticia que no se ve compensada con el gasto energético, debido a un déficit de actividad física.

La prevalencia del sobrepeso y la obesidad está aumentando de forma tan alarmante en los países occidentales que algunos estudios ya hablan de este fenómeno en términos de epidemia ${ }^{13,48}$. No debemos perder de vista las consecuencias que la pérdida de actividad física en la vida diaria, el sobrepeso y la obesidad tienen en la economía de los países que padecen este modo de vida, y las fuertes tasas de morbilidad y mortalidad prematuras que estas acarrean. Por ejemplo, en los EEUU, el coste directo generado por la inactividad y la obesidad consume el $9,4 \%$ del gasto médico anual ${ }^{21}$, mientras que en España, esta cifra ronda ya el $7 \%$.

Aunque los estudios que analizan la asociación entre la AC y el NAF en adultos obtienen resultados concluyentes, no suele ocurrir lo mismo en los estudios realizados con adolescentes. Parece que, en muchos casos, al igual que ocurría con la PA, en los sujetos jóvenes aún no ha dado tiempo a 
que se manifiesten los efectos de la inactividad física en su composición corporal. Sin embargo, creemos que son necesarias investigaciones posteriores que informen de la evolución de esta asociación entre los adolescentes y, mediante estudios longitudinales, de la evolución de la composición corporal a lo largo de los años posteriores a la adolescencia en función del NAF.

\section{BIBLIOGRAFÍA}

1. Krekoukia M, Nassis GP, Psarra G, Skenderi K, Chrousos GP y Sidossis LS. Elevated total and central adiposity and low physical activity are associated with insulin resistance in children. Metabolism. 2007; 56: 206-13.

2. Perula de Torres LA, Lluch C, Ruiz Moral R, Espejo Espejo J, Tapia G y Mengual Luque P. Prevalencia de actividad física y su relación con variables sociodemográficas y ciertos estilos de vida en escolares cordobeses. Rev Esp Salud Publica. 1998; 72: 233-44.

3. Boreham CA, Twisk J, Savage M J (1997). Physical activity, sports participation, and risk factors in adolescents. Med Sci Sports Exerc. 1997; 29: 78893.

4. Raitakari OT, Taimela S, Porkka KVK, Telama R, Välimäki I, Akerblom HK y Viikari JSA. Associations between physical activity and risk factors for coronary heart disease: the cardiovascular risk in young finns study. Med Sci Sports Exerc. 1997; 29: 1055-61.

5. Pate RR, HeathGW, Dowda M y Trost SG. Associations between physical activity and other health behaviors in a representative sample of U.S. adolescents. Am J Public Health. 1996; 86: 1577-581.

6. Janz KF, Dawson JD y Mahoney LT. Tracking physical fitness and physical activity from childhood to adolescence: the Muscatine study. Med Sci Sports Exerc. 2000; 32: 1250-7.

7. National Center for Chronic Disease Prevention and Health Promotion; Centers for Disease Control and Prevention. Guidelines for school and community programs to promote lifelong physical activity among young people. J Sch Health. 1997; 67: 202-19.

8. Katzmarzyk PT y Janssen I. The economic cost associated with physical inactivity and obesity in
Canada: an update. Can J Appl Physiol. 2004; 29: 90-107.

9. Gavarry O y Falgairette G. L'activité physique habituelle au cours du développement. Can J Appl Physiol. 2004; 29: 201-214.

10. Cantera MA y Devís. J. La promoción de la actividad física relacionada con la salud en el ámbito escolar. Implicaciones y propuestas a partir de un estudio realizado entre adolescentes. Apunts. 2000; 6: 54-62.

11. Savage MP y Scott LB. Physical activity and rural middle school adolescents. J Youth Adolesc. 1998; 27: 245-253.

12. Cale L y Almond L Physical activity levels of secondary-aged children: a review. Health Educ J. 1992; 51: 192-7.

13. Banegas JR. Epidemiología de la hipertensión arterial en España. Situación actual y perspectivas. Hipertensión. 2005; 22: 353-62.

14. García del Pozo J, Ramos Sevillano E, De Abajo FJ y Mateos Campos R. Utilización de antihipertensivos en España (1995-2001). Rev Esp Cardiol. 2004; 57: 241-9.

15. Kelley GA y Kelley KS. Exercise and resting blood pressure in children and adolescents: a meta-analysis. Pediatr Exerc Sci. 2003; 15: 83-97.

16. Strong WB, Malina RM, Blimkie CJR, Daniels SR, Dishman RK, Gutin B, Hergenroeder AC, Must A, Nixon PA, Pivarnik JM, Rowland T, Trost S y Trudeau F. Evidence Based Physical Activity for School-age Youth. J Pediatr. 2005; 146: 73237.

17. Hawkins SA, Cockburn MG, Hamilton AS y Mack TS. An estimate of physical activity prevalence in a large population-based cohort. Med Sci Sports Exerc. 2004; 36: 253-60.

18. Vriz O, Mos L, Frigo G, Sanigi C, Zanata G, Pegoraro F y Palatini P. Effects of physical exercise in clinic and 24-Hour ambulatory blood pressure in young subjects with mild hypertension. J Sports Med Phys Fitness. 2002; 42: 83-8.

19. López Calbet A, Dorado C y Chavarren J. (1996). Evaluación de la composición corporal mediante absorciometría fotónica dual de rayos X. En: Consejo Superior de Deportes, editor. Métodos de Estudio de Composición Corporal en Deportistas. Madrid: Ministerio de Educación y Cultura. Consejo Superior de Deportes; 1996. p. 55-79. 
20. Caspersen CJ, Nixon PA y Durant RH. Physical activity epidemiology applied to children and adolescent. Exerc Sport Sci Rev. 2000; 26: 241-403.

21. Grundy SM, Blackburn G, Higgins M, Lauer R, Perri MG y Ryan D. Physical activity in the prevention and treatment of obesity and its comorbidities: evidence report of independent panel to assess the role of physical activity in the treatment of obesity and its comorbidities. Med Sci Sports Exerc. 1999; 31: 1493-500.

22. Nelson MC, Neumark-Stzainer D, Hannan PJ, Sirard JR y Story M Longitudinal and secular trends in physical activity and sedentary behavior during adolescence. Pediatrics. 2006; 118: 1627 34.

23. Pate RR, Pfeiffer KA, Trost SG, Ziegler P y Dowda M. Physical Activity among children attending preschools. Pediatrics. 2004; 114: 1258-63.

24. Guo SS y Chumlea WC. Tracking of body mass index in children in relation to overweight in adulthood. Am. J. Clin. Nutr. 1999; 70: S145-8.

25. Albright AL y Stern JS. Adipose tissue. Encyclopedia of Sports Medicine and Science. Internet Society for Sport Science (1998, mayo). Disponible en: Http://www.sportsci.org.

26. Guidelines Subcommittee.1999 World Health Organization-International society of hypertension guidelines for the management of the hypertension. J Hypertens. 1999; 17: 151-83.

27. Aaron DJ y Kriska AM. Modifiable activity questionnaire for adolescents. Med Sci Sports Exerc. 1997; 29 (1 Supl 2): 79-82.

28. Cordente Martínez CA. Estudio epidemiológico del nivel de actividad física y de otros parámetros de interés relacionados con la salud bio-psicosocial de los alumnos de E.S.O. del Municipio de Madrid [Tesis doctoral]. Toledo: Universidad de Castilla-La Mancha; 2006. Disponible en: http://www.cafyd.com/tesis 12cordente.pdf

29. Van Mechelen W, Twisk JWR, Berheke Post G, Snel J y Kemper HCG. Physical activity of young people: the Amsterdam longitudinal growth and health study. Med Sci Sports Exerc. 2000; 32: 1610-17.

30. Divisón Garrote JA. Medidas de presión arterial de forma semiautomática en el diagnostico de la hipertensión arterial. Comparación con otros métodos diagnósticos y estudio de su variabilidad y reproducibilidad [Tesis Doctoral]. Madrid: Universidad Autónoma de Madrid; 1998.
31. Grupo Cooperativo Español para el Estudio de los Factores de Riesgo Cardiovascular en la Infancia y Adolescencia. Factores de riesgo cardiovascular en la infancia y adolescencia en España. Estudio RICARDIN II: Valores de referencia. An Esp Pediatr. 1995; 43: 11-17.

32. Aragonés Clemente MT, Casajús Mallen JA, Rodríguez Guisado F, Cabañas Armesilla MD. Protocólo de medídas antropométricas. En: Esparza Ros F, editor. Manual de Cineantropometría. Monografías FEMEDE. Madrid: FEMEDE; 1993 p. 35-66.

33. Weiner JS y Lourie JA. Human biological. A guide to field methods. International Biological Programme (handbook $n^{\circ}$ 9). Oxford: Blackwell scientific; 1969.

34. Pacheco Del Cerro JL 1996. Valoración antropométrica de la masa grasa en atletas de elite. En Consejo Superior de Deportes, editor. Métodos de estudio de composición corporal en deportistas. Madrid: Ministerio de Educación y Cultura-Consejo Superior de Deportes 1996. p. 27-54.

35. Porta J, Galiano D, Tejedo A, González JM 1993. Valoración de la composición corporal. utopías y realidades. En: Esparza Ros F., editor. Manual de Cineantropometría. Monografías FEMEDE. Madrid: FEMEDE 1993. p. 113-170.

36. Cantera MA y Devís J. Physical activity levels of secondary school spanish adolescents. Eur J Physical Educ. 2002; 5: 28-44.

37. Aaron DJ y Laporte RE (1997). Physical activity, adolescence and health: an epidemiological perspective. Exerc Sport Sci Rev. 1997; 25: 391-405.

38. Dencker M, Thorsson O, Karlsson MK, Linden C, Svensson J, Wollmer P y Andersen LB. Daily physical activity in Swedish children aged 8-11 years. Scand J Med Sci Sports. 2006; 16: 252-7.

39. Ministerio de Sanidad y Consumo. Encuesta Nacional de Salud. Madrid: Ministerio de Sanidad y Consumo; 2001.

40. Michaud-Tomson L, Davidson M y Cuddihy TF. Walk to school does it make a difference in children's physical activity levels? ACHPER Healthy Lifestyles Journal 2004; 50: 16-24.

41. Riddoch CJ, Andersen LB, Wedderkopp N, Harro M, Klasson-Heggebo L, Sardinha LB, Cooper AR y Ekelund U. Physical activity levels patterns of 9and 15-years-old european children. Med Sci Sports Exerc. 2004; 36: 86-92. 
42. Welk GJ, Schaben JA y Shelley M. Physical activity and physical fitness in children schooled at home and children attending public schools. Pediatr Exerc Sci. 2004; 16: 310-23.

43. Riddoch CJ, Bo Andersen L, Wedderkopp N, Harro M, Klasson-Heggebo L, Sardinha, LB, Cooper AR y Ekelund U. Physical activity levels and patterns of 9- and 15-yr-old European children. Med Sci Sports Exerc. 2004; 36: 86-92.

44. Boreham C, Twisk J, Neville C, Savage M, Murray L. y Gallagher A. Associations between physical fitness and cardiovascular risk factors in young adulthood: the Northern Ireland young hearts project. Int J Sports Med 2002; 23 [1 suplemento 2]: 22-6.

45. Paulus D, Saint-Remy A y Jeanjean M. Blood pressure during adolescence: a study among belgian adolescents selected from a high cardiovas- cular risk population. Eur J Epidemiol. 1999; 15: 783-90

46. Soler MB, Gil A y Rey J. Estudio epidemiológico de la tensión arterial en una población escolar. Aten Primaria. 1992; 9: 212-4.

47. De Vito E, La Torre G, Langiano E, Berardi D y Ricciardi G. Overweight and obesity among secondary school children in central Italy. Eur J Epidemiol. 1999; 15: 649-54.

48. Tudor-Locke C y Myers AM. Challenges and opportunities for measuring physical activity in sedentary adults. Sports Med. 2001; 31: 91-100.

49. Vázquez Sánchez R y López Alemany JM. Los costes económicos de la obesidad alcanzan el 7\% del gasto sanitario [citado en 2003]. http://www.economiadelasalud.com/Ediciones/03/PDF/03Analisis_Costes.pdf. 
